\title{
Manifestaciones neurológicas y COVID-19
}

\section{Neurologic manifestations and COVID-19}

Viroj Wiwanitkit*

D.Y. Patil University, Departamento de Medicina Comunitaria, Pune, India

En el artículo de Jiménez-Ruiz et al., titulado "Manifestaciones neurológicas de COVID-19", se llegó a la conclusión de que "debemos tener en cuenta las complicaciones infecciosas y no infecciosas que afectan el sistema nervioso como la encefalitis, las convulsiones, el síndrome de Guillain-Barré, la encefalomielitis diseminada y la leucoencefalitis hemorrágica, que son trastornos que pueden ocurrir durante o después de las infecciones virales. Un síntoma neurológico podría ser la primera manifestación de COVID-19". 'De hecho, COVID-19 puede dar lugar a numerosas presentaciones atípicas, lo cual dificulta el diagnóstico; ${ }^{2}$ sin embargo, el problema neurológico concurrente representa una complicación importante. Un paciente con COVID-19 podría tener un trastorno neurológico subyacente, como epilepsia o infarto cerebrovascular, lo que podría derivar en una evolución clínica más compleja. Además, existe la posibilidad de otro trastorno médico concurrente, como la coinfección, que puede desencadenar trastornos neurológicos en pacientes con COVID-19. Un buen ejemplo es el dengue concurrente y la infección por COVID-19. ${ }^{3}$

\section{Bibliografía}

1. Jiménez-Ruiz A, García-Grimshaw M, Ruiz-Sandoval JL. Manifestaciones neurológicas de COVID-19. Gac Med Mex. 2020;156:257.

2. Tin SS, Wiwanitkit V. Uncommon atypical presentations of COVID-19: Important and should not be under recognized! J Health Med Sci Res. 2020;38:153-158.

3. Magalhaes T, Chalegre KDM, Braga C, Foy BD. The endless challenges of arboviral diseases in Brazil. Trop Med Infect Dis. 2020;5:E75. CC BY-NC-ND (http://creativecommons.org/licenses/by-nc-nd/4.0/). 